\title{
Development of a novel model of driver-vehicle steering control incorporating sensory dynamics
}

\author{
C.J. Nash \\ University of Cambridge, United Kingdom \\ D.J. Cole \\ University of Cambridge, United Kingdom
}

\begin{abstract}
A novel model of driver steering control is proposed, incorporating models of the driver's sensory dynamics and limitations. The model is based on the hypothesis that the driver's steering strategy minimises an internal cost function optimally based on the noisy, delayed information received from the sensory systems. Published results from experiments carried out on pilots were used to identify parameter values for the new model, and to assess the validity of the new modelling approach. The new model was found to fit the results very well, with variance accounted for (VAF) values greater than $90 \%$ for all but one trial. The model was found to fit the results almost as well with a single fixed set of parameter values as with separate parameter values for each trial, indicating that a fixed-parameter model is able to predict variations in control behaviour under different conditions.
\end{abstract}

Keywords: driver-vehicle dynamics, control, steering, sensory dynamics, modelling, optimal control, model identification

\section{INTRODUCTION}

There are a large number of models of driver steering control available, as reviewed comprehensively by Plöchl \& Edelmann (2007). However, very few of these consider the driver's sensory dynamics, which may have an important role in shaping the observed steering strategy. There is a large body of research into human sensory systems available, however most of this is based on measurements taken under tightly controlled conditions. Bigler (2013) attempted to use these results to build a driver model incorporating sensory dynamics, however the model did not match experimental results well, indicating that some parameter values may have been chosen incorrectly.

Studies have shown that sensory noise levels may be different during an active control task such as driving compared with the isolated conditions under which sensory systems have generally been studied (Samji \& Reid 1992, Zaichik et al. 1999, Rodchenko et al. 2000, Valente Pais et al. 2012). During a steering control task, a variety of different sensory stimuli are presented to the driver at once, and the driver's attention must be distributed between these and the control task. This may be one of the reasons that the model of Bigler (2013) did not agree well with measured results. Therefore it is proposed that a parametric identification procedure could be used to investigate how sensory systems perform during driving. A novel model of driver steering control has been developed based on models of drivers' sensory dynamics, in order to develop a deeper understanding of how sensory information is used by drivers to control a vehicle and to predict how drivers will steer in different situations.

Researchers at TU Delft have published results from tracking tasks carried out by pilots in a flight simulator (Zaal et al. 2009a). These tasks are very similar to the steering control task carried out by drivers, therefore the results have been used to identify parameter values for the new driver model, adapted slightly to match the pilot tracking task. The agreement between the new model 
and the results of Zaal et al. (2009a) was quantified in order to assess the modelling and identification methodologies. This information can then be used when designing future experiments using drivers in order to validate the model for a driver steering control task.

The new model of driver steering control is presented in Section 2. A description of the experimental data and procedure used for identification of parameter values is given in Section 3, then the results of this are presented and discussed in Section 4. The main conclusions drawn from the work are summarised in Section 5.

\section{NEW STEERING CONTROL MODEL}

It has been suggested that driver steering control consists of two levels of control behaviour, a feedforward level and a feedback level (Donges 1978). The feedforward level involves looking at the road ahead to follow a target trajectory (for example the middle of the lane or a 'racing line'). The feedback level involves compensating for disturbances on the vehicle, which may be a result of gusts of wind, bumps in the road, nonlinear vehicle dynamics or uncertainties introduced by the driver.

A new model of driver steering control has been developed combining these two levels, incorporating the driver's sensory dynamics in order to provide a physical explanation for observed control behaviour. The model is based on the hypothesis that drivers steer in order to minimise the deviation of the vehicle from a target trajectory, and that they do so optimally on average based on the noisy information they get from their sensory systems. The structure of the new model is shown in Figure 1. It is based upon the linear quadratic Gaussian (LQG) framework, which combines a linear quadratic regulator (LQR) with a Kalman filter. Used together these give a control system which calculates a mathematically optimal input to control the plant based on noisy sensory measurements and knowledge of previous plant inputs. The plant contains the dynamics of the system, including human sensory dynamics and neuromuscular dynamics. The most important sensory systems for driving are thought to be the visual and vestibular systems, which are included in the model. Other sensory information may be obtained from torque feedback through the steering wheel or pressure and force sensors within the body, however for simplicity these have been omitted.

\subsection{Plant}

The plant is shown in more detail in Figure 2. The target $f_{t}$ and disturbance $f_{d}$ are known as forcing functions, as they can be added into the system during an experiment in order to elicit responses from the driver which can be measured to investigate the driver's steering control strategy. They are modelled as filtered Gaussian white noise, since the LQR controller and Kalman

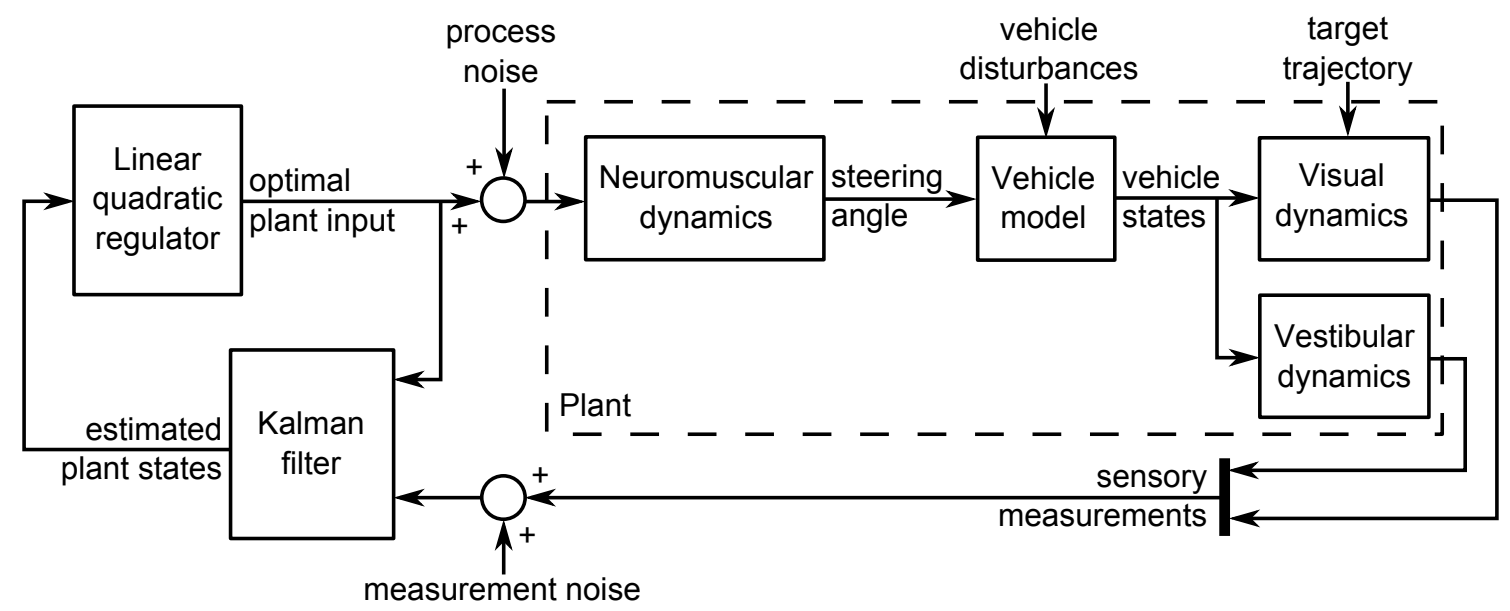

Figure 1. Structure of new model of driver steering behaviour. The plant consists of models of the vehicle dynamics, human neuromuscular dynamics and sensory systems. An LQR controller is used to compute the optimal plant input (Sharp \& Valtetsiotis 2001, Cole, Pick \& Odhams 2006), and a Kalman filter is used to find an optimal state estimate in the presence of Gaussian measurement and process noise. 
filter require all unknown inputs to have a Gaussian distribution. The forcing function distributions $H_{f d}(s)$ and $H_{f t}(s)$ are therefore included in the dynamics of the plant. The vestibular system consists of two sets of sensory organs located in the inner ear, the otoliths which are sensitive to translational accelerations and the semi-circular canals (SCCs) which are sensitive to angular velocity. The visual system can be modelled as a 'preview' controller (Sharp \& Valtetsiotis 2001, Cole, Pick \& Odhams 2006), as shown in Figure 3. The driver looks ahead and 'previews' the lateral displacements of the target path every time step up to a 'preview horizon'. The plant also contains descriptions of the vehicle dynamics and the driver's neuromuscular dynamics, which act as a filter on the steering inputs to the vehicle due to the dynamic properties of the driver's arms. The model is implemented in discrete time, so that the time delays can be modelled explicitly using a shift register (Pick 2004). This is a matrix which 'shifts' the delayed variable down one state at each time step until the desired time delay has been achieved.

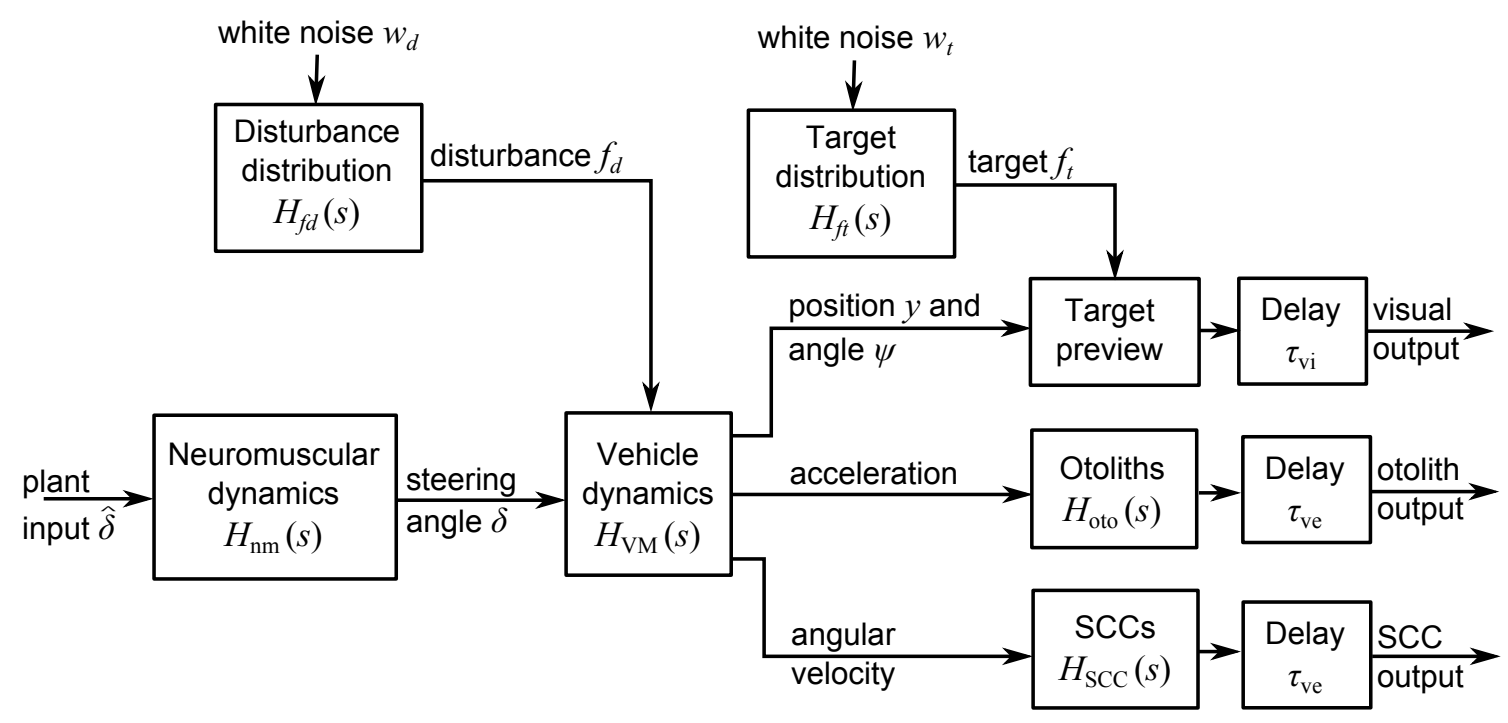

Figure 2. Dynamics of the plant in the new driver steering model. The target and disturbance are input as white noise $w_{t}$ and $w_{d}$, which is filtered by their distributions $H_{f t}(s)$ and $H_{f d}(s)$. The vehicle motion is sensed by the visual system using a target preview model (shown in Figure 3) as well as the otoliths and the semi-circular canals (SCCs). The perceived motion is delayed by visual and vestibular delays $\tau_{v i}$ and $\tau_{v e}$ to represent latencies in the brain of the driver.

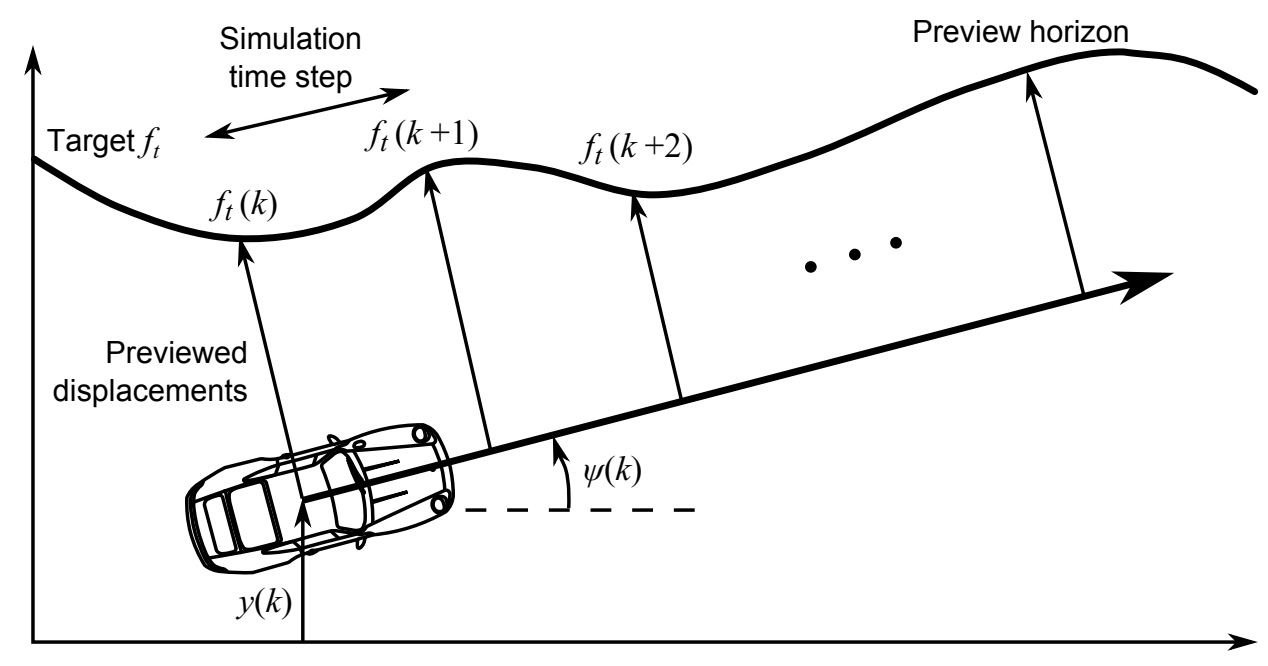

Figure 3. Model of 'preview' of upcoming target path (Sharp \& Valtetsiotis 2001, Cole, Pick \& Odhams 2006). The driver takes measurements of the lateral displacement between the target path and a vector projected straight ahead of the vehicle. Measurements are taken at multiples of the simulation time step up to a preview horizon. 


\subsection{LQR controller and Kalman filter}

A linear quadratic regulator (LQR) is an optimal controller which minimises a quadratic cost function $J$. For a linear plant, the optimal LQR solution is time-invariant and consists of a vector of gains acting on the states of the plant. The optimal solution is not affected by white noise disturbances, which is why the target and disturbance must be input to the plant as white noise. The cost function takes account of the trade-off the driver must make between the amount of effort they put into steering and the accuracy with which they follow the target. This is achieved by including costs on the target following error $\left(y-f_{t}\right)$ and the plant input $\hat{\delta}$, multiplied by a weighting factor $q_{\delta}$ to balance the relative importance of the two costs:

$$
J=\sum_{k=0}^{\infty}\left(q_{\delta} \hat{\delta}(k)^{2}+\left\{y(k)-f_{t}(k)\right\}^{2}\right)
$$

The LQR controller calculates a vector of gains which act on the plant states to give the optimal plant input $\hat{\delta}$, however the driver does not have access to all of the plant states. The driver only takes measurements of the plant outputs, and these are perturbed by measurement noise as shown in Figure 1. The driver also knows the computed plant input, although process noise is added to this input to represent uncertainties in the driver's calculation of the optimal steering strategy. The Kalman filter finds an optimal state estimate under such conditions, and for a linear invariant system with stationary Gaussian noise an optimal linear invariant Kalman filter can be found.

\subsection{Transfer functions for sensory and neuromuscular dynamics}

Transfer functions for the otoliths and semi-circular canals (SCCs) have been studied extensively in the literature, based on models of the physical dynamics of the organs and experiments carried out on humans and primates. A number of these were reviewed by Telban and Cardullo (2005), and the following transfer functions were suggested:

$$
\begin{aligned}
& H_{\text {oto }}(s)=\frac{0.4(1+10 s)}{(1+5 s)(1+0.016 s)} \\
& H_{\text {SCC }}(s)=\frac{5.73\left(80 s^{2}\right)}{(1+80 s)(1+5.73 s)}
\end{aligned}
$$

The vestibular system has been found to perform similarly in all motion axes, so these transfer functions can be used for motion in any direction.

The dynamics of a driver's neuromuscular system have also been studied extensively, and detailed models of open and closed loop neuromuscular responses have been developed. The focus of this study is not on neuromuscular dynamics, therefore a simple neuromuscular model was used. Pick \& Cole (2007) used a shaker to apply random torque disturbances to a steering wheel and measured the response of the driver's arm muscles. They found that the results could be approximated by a second-order transfer function:

$$
H_{\mathrm{nm}}(s)=\frac{\omega_{\mathrm{nm}}^{2}}{s^{2}+2 \omega_{\mathrm{nm}} \zeta_{\mathrm{nm}} s+\omega_{\mathrm{nm}}^{2}}
$$

Different values of the natural frequency $\omega_{\mathrm{nm}}$ and damping factor $\zeta_{\mathrm{nm}}$ were found by Pick \& Cole (2007) depending on whether the driver had tensed or relaxed arms. During a driving task the driver may relax, tense or partially tense their arms depending on the conditions. Therefore the values of $\omega_{\mathrm{nm}}$ and $\zeta_{\mathrm{nm}}$ were found using an identification procedure to investigate how their values change under different conditions.

\section{IDENTIFICATION OF MODEL PARAMETER VALUES}

A new model of driver steering control is presented in Section 2, based on models of the driver's physical limitations and sensory dynamics. However the performance of the model depends on noise magnitudes, time delays and a cost function weight which were not known. An identification procedure was used to find parameter values which gave the best possible agreement between predicted and measured steering behaviour. The performance of the new model was then evaluated by quantifying the agreement between the experimental results and the best model prediction. 
Researchers at TU Delft have measured pilots' control signals in combined target-following and disturbance-rejection tasks carried out in a flight simulator and fitted the results to a simple parametric model consisting of gains, time delays and lead and lag terms (Zaal et al. 2009a). Pitch tracking tasks for pilots are very similar to steering tasks for drivers, therefore the results published by Zaal et al. (2009a) were used to reconstruct the measured pilot control signals for comparison with predictions made by the new model. The results of this comparison were used to validate the new modelling approach, and they will be used in future work to inform the design of a new experiment carried out in a driving simulator.

\subsection{Pilot pitch tracking experiment results}

Zaal et al. (2009a) measured the performance of pilots in a combined target-following and disturbance-rejection task carried out in a flight simulator. The task was carried out in the pitch axis alone, with no translational motion applied to the pilots. The structure of the experiment is shown in Figure 4. Zaal et al. (2009a) devised a simple pilot model to quantify the control strategy of the pilots. The model was split into two transfer functions, a visual response acting on the tracking error $e=\left(f_{t}-\theta\right)$ and a motion response acting on $\theta$. These transfer functions were constructed from gains, time delays and lead and lag terms which were found by Zaal et al. (2009a) using an identification procedure to describe the pilots' control behaviour in each trial. This model gave an accurate fit to the experimental data, with variance accounted for (VAF) values greater than $85 \%$ in each trial, however it is an empirical model and does not provide a physical basis for the pilots' actions in the same way as the new model.

Eight separate trials were carried out by Zaal et al. (2009a), varying the relative powers of the target and disturbance forcing functions. The conditions varied from disturbance-only in trial 1 to target-only in trial 8 , however the combined power of the target and disturbance signals was the same in all trials. Zaal et al. (2009a) published the identified parameter values for each trial using their empirical model, and these were used to reconstruct the control signals from their experiment. These reconstructed control signals were then used to identify parameter values for the new model to investigate how well the new model can match the results from this experiment. The reconstructed control signals do not contain any of the noise from the original measurements, however they also do not contain any variations in control signal which were not captured by the empirical model, therefore it is not possible to compare the performance of the two models directly.

The tracking task carried out by pilots in the study of Zaal et al. (2009a) is analogous to the steering control task carried out by drivers as shown in Figure 1, with the control signal $u$ replacing the steering angle $\delta$, the aeroplane dynamics $H_{a}(s)$ replacing the vehicle model $H_{\mathrm{VM}}(s)$ and the pitch angle $\theta$ replacing the lateral displacement $y$. The aeroplane dynamics were given by:

$$
H_{a}(s)=-10.6189 \frac{s+0.9906}{s\left(s^{2}+2.756 s+7.612\right)}
$$

The target pitch angle was displayed to the pilots as a line on a screen, with the current pitch angle shown by a crosshair. The pilots were therefore not able to preview future values of the

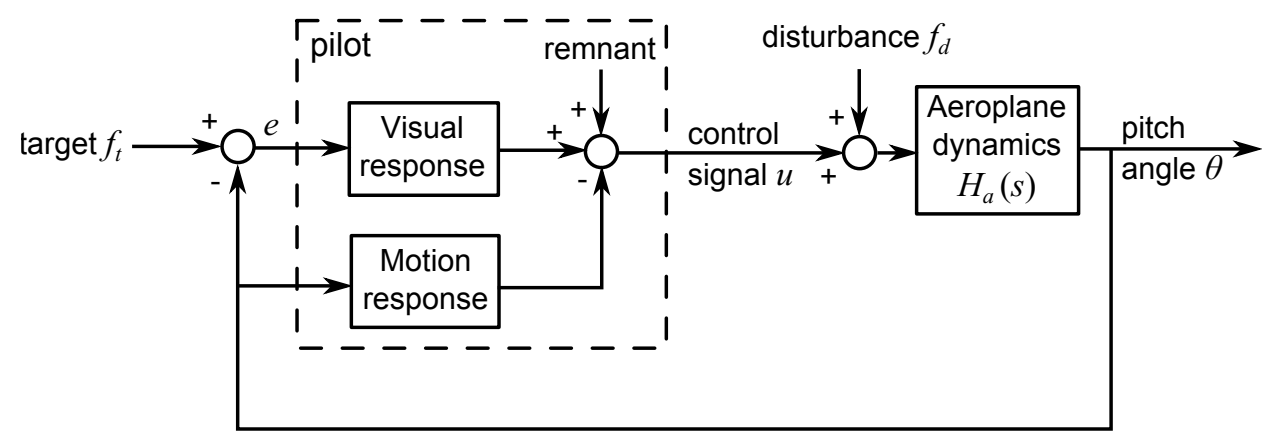

Figure 4. Structure of experiment carried out by Zaal et al. (2009a). The pilots were instructed to follow the target pitch angle as closely as possible, while disturbances were added to the control signal $u$. The pilot was modelled by Zaal et al. (2009a) as two transfer functions, the visual response and the motion response, which were made up of gains, time delays and lead and lag terms. 


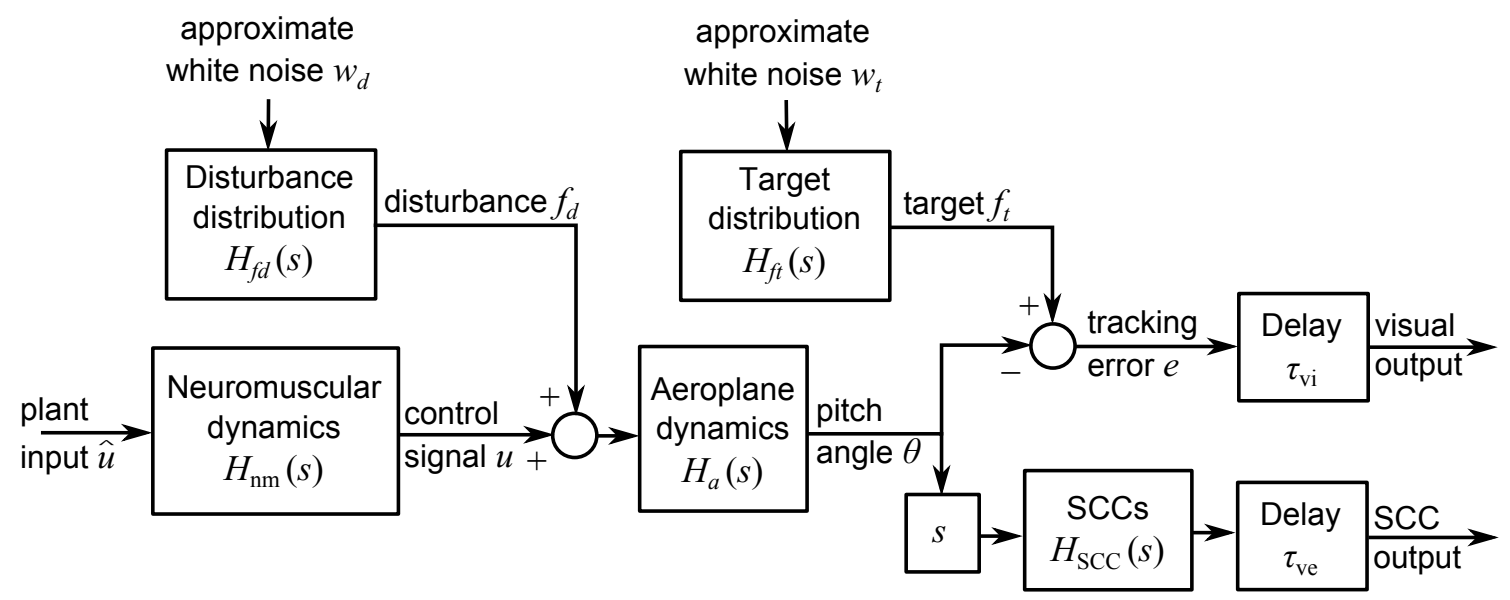

Figure 5. Adapted plant to model the pilot pitch tracking task carried out by Zaal et al. (2009a). The steering angle $\delta$ is replaced with the control signal $u$, and the disturbance $f_{d}$ is added directly to this. Instead of the 'preview' model of the driver's visual system, the pilot's visual system directly measures the tracking error $e$.

target like drivers, but instead their visual dynamics were modelled as a direct measurement of the tracking error $e$. The plant for the new driver model was adapted as shown in Figure 5 to match the pilot tracking task so that the results of Zaal et al. (2009a) could be used to test the validity of the new model.

In most respects the experiment of Zaal et al. (2009a) matches the structure of the new model very well. However one area where they differ is in the definition of the target and disturbance forcing functions. In the new model they must be defined as filtered white noise in order to find the optimal LQR and Kalman filter solutions, however in the experiment of Zaal et al. (2009a) the forcing functions were formed from sums of sinusoids. The amplitudes of these sinusoids followed the distributions:

$$
\begin{aligned}
& H_{f t}(s)=\left(\frac{1+0.1 s}{1+0.8 s}\right) \\
& H_{f d}(s)=\left(\frac{1+0.1 s}{1+0.8 s}\right) H_{a}(s)^{-1}
\end{aligned}
$$

The disturbance was added to the control signal before the aeroplane dynamics, therefore the distribution of the disturbance on the pitch angle $\theta$ was the same as the distribution of the target.

Since the distributions of the target and disturbance signals are known, the sum-of-sinusoid signals given by Zaal et al. (2009a) can be filtered by the inverse of these distribution to give approximate white noise inputs $w_{t}$ and $w_{d}$. However, for the optimal Kalman filter solution to be found, their RMS magnitudes $W_{t}$ and $W_{d}$ must be known. This presents a problem, as the power of the sum-of-sinusoids signals is concentrated at the sinusoid frequencies, whereas filtered white noise has a smooth power spectrum. This means that if the magnitudes $W_{t}$ and $W_{d}$ were chosen to match the RMS values of $w_{t}$ and $w_{d}$, the magnitudes of the $f_{t}$ and $f_{d}$ signals would be different to the magnitudes of true filtered white noise signals. The values of $W_{t}$ and $W_{d}$ were chosen so that the magnitudes of $f_{t}$ and $f_{d}$ found by filtering true white noise were the same as the magnitudes of $f_{t}$ and $f_{d}$ found from the sums of sinusoids. This means that the magnitudes of the target and disturbance signals in the pilot model are correct, however the differing power spectra will inevitably lead to mismatches between the assumed and actual magnitudes elsewhere in the model.

\subsection{Identification procedure}

In the case of the pilot tracking task, the control strategy of the new model can be described by eight parameters. These are the cost function weight $q_{u}$ which affects the trade-off between control effort and tracking performance, the RMS magnitudes $V_{e}$ and $V_{\omega}$ of the Gaussian measurement noise added to the visual and SCC outputs respectively, the RMS magnitude $W$ of the process 
noise added to the plant input, visual and vestibular delays $\tau_{\mathrm{vi}}$ and $\tau_{\mathrm{ve}}$ and the natural frequency $\omega_{\mathrm{nm}}$ and damping factor $\zeta_{\mathrm{nm}}$ for the pilot's neuromuscular dynamics. An identification procedure was used to find the values of these parameters which gave the best fit to the experimental results reconstructed from Zaal et al. (2009a).

A detailed overview of parametric identification procedures was given by Ljung (1999). There are two main types of identification, direct identification where the system (in this case the pilot or driver) is simulated in open loop, and indirect identification where the system is simulated in closed loop. Because the aeroplane dynamics are known indirect identification can be used, which should result in lower bias. The identification procedure then becomes a task of minimising the modelling error, defined as the difference between the measured and modelled control signals. Ljung (1999) showed that if the system contains noise, the modelling error should be filtered by the inverse of the noise model to reduce bias in the results. This was not done in this case as the reconstructed control signals were found from a linear simulation so were free of noise.

The minimisation task is not an easy one, as it must be carried out over eight parameters and the search space may contain several local minima. A genetic algorithm was therefore used to find an initial estimate of the optimal set of parameter values, starting with a population of 100 random solutions and using principles of natural selection to converge towards the global minimum (Zaal et al. 2009b). The best solution found by the genetic algorithm was then used as the starting point for the Matlab function fmincon, which uses a gradient search method to converge more efficiently towards the minimum.

Firstly, separate parameter values were identified to fit the results of each individual trial as closely as possible. A single set of parameter values was then identified to fit the results of all of the trials, to determine how well the new model can predict the variations in control behaviour observed under different conditions. Similarly to the identification process carried out by Zaal et al. (2009a), the modelled control signal was found for each set of parameter values by simulating each $90 \mathrm{~s}$ trial, but only the last $81.92 \mathrm{~s}$ were considered when comparing the reconstructed and modelled control signals.

\section{RESULTS AND DISCUSSION}

The procedure described in Section 3.2 was used to find parameter values to fit the results of Zaal et al. (2009a). The agreement between the reconstructed control signal $u_{\text {exp }}$ and the control signal $u_{\text {model }}$ predicted by the new model was quantified by calculating the variance accounted for (VAF), which measures the percentage of the variance of the pilot control signal which is predicted by the model:

$$
\mathrm{VAF}=\left(1-\frac{\sum_{k}\left\{u_{\exp }(k)-u_{\text {model }}(k)\right\}^{2}}{\sum_{k}\left\{u_{\text {exp }}(k)\right\}^{2}}\right) \times 100 \%
$$

The resulting VAF values are shown in Figure 6. In all trials except trial 8 the VAF values are greater than $90 \%$, showing that the new model explains the results very well. The VAF is significantly lower for trial 8, which may indicate that the model fits less well for pure target-following tasks. The VAF values found using a single set of parameter values to fit all the trials are very close to the values found using separate sets of parameter values for each trial, which shows that a fixed-parameter model can predict pilot control behaviour under a range of conditions.

The pilot control signals reconstructed from the results of Zaal et al. (2009a) are compared with the signals predicted by the model in Figure 7, for the trials with the highest (trial 5) and lowest (trial 8) VAFs. The first $8 \mathrm{~s}$ of each trial do not fit particularly well, which is most likely a result of Zaal et al. (2009a) ignoring the first $8 \mathrm{~s}$ of each trial when identifying parameter values for their empirical model. After this initial transient period the signals match very well in trial 5, with some small modelling errors in the higher frequency components. The agreement of the two signals for trial 8 is not as good as for trial 5, however the model still captures the majority of the variation seen in the control signal. Without having access to the original data it is not possible to assess what proportion of the modelling error can be attributed to the new model and what proportion can be attributed to the model used by Zaal et al. (2009a).

The identified parameter values for each trial are shown in Figure 8. There are some clear trends in the identified values, although they are not always followed by trial 8 which did not fit the experimental results as well. The noise parameters $V_{e}, V_{\omega}$ and $W$ all increase with increasing 


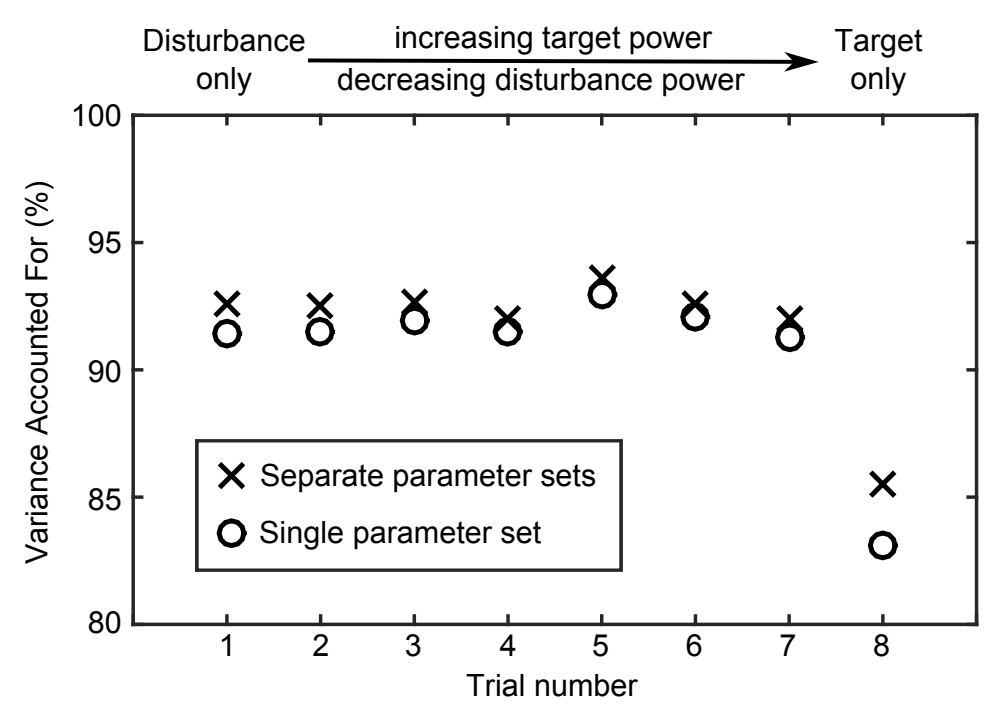

Figure 6. Variance accounted for (VAF) values found when fitting the predictions of the new model to control signals reconstructed from the published results of Zaal et al. (2009a). Values found for separate sets of parameter values optimised to fit each individual trial are shown by crosses, whereas values found using a single set of parameter values optimised to fit all of the trials are shown by circles.

target power, although without access to the original noisy data it is not possible to tell whether the noise level actually increased or whether this is simply a shift in the weightings of the Kalman filter. The vestibular delay $\tau_{\text {ve }}$ also increases with target power, indicating that the pilot may use their vestibular system primarily to respond to disturbances. The visual delay is shorter than the vestibular delay in all trials. The cost weight $q_{u}$ and neuromuscular frequency $\omega_{\mathrm{nm}}$ both decrease with increasing target power, whereas the neuromuscular damping $\zeta_{\mathrm{nm}}$ appears to be largest when the target and disturbance powers are equal (trial 5).

The single set of parameter values identified to fit all of the trials is shown by the horizontal lines in Figure 8. They are in general close to the separate parameter values, and all lie within the range of the values found for the separate trials. The VAF values in Figure 6 show that, although the variations in parameter values seen in Figure 8 appear to be significant, they do not have a large effect on the predictions of the model. The single set of parameter values fits the results almost as well as the separate parameter values, so it is not necessary to model all of these variations in parameter values to get a good description of the pilots' control behaviour.

The fact that the new model fits the results so well is very encouraging, and indicates that the assumptions made by the model are reasonable. The model does not fit as well for the pure target-following task, however the visual perception of the target is one of the main areas where the pilot and driver models differ, so the accuracy of this part of the model is less important for validating the driver steering control model. The experimental results are matched almost as well using a single set of parameter values fixed across the trials as with different parameter values for each trial, indicating that the new model is able to predict pilot tracking behaviour under various conditions. The model seems to be a reasonable description of pilot tracking behaviour, however further work is necessary to validate the model for driver steering behaviour. The results of this investigation can be used to guide the design of new experiments using drivers to provide data which can be used to identify parameter values for the driver steering control model.

\section{CONCLUSIONS}

A new model of driver steering control has been developed based on models of human sensory dynamics and the hypothesis that drivers steer close to optimally based on their sensory limitations. Driver and pilot control tasks are very similar, therefore results from an experiment carried out in a flight simulator (Zaal et al. 2009a) have been used to investigate the validity of the new model. The model has been found to agree closely with the experimental results, with variance accounted for (VAF) values greater than $90 \%$ in all except one trial. The model didn't fit as well for a pure target-following trial, indicating that the model may not describe pilot target-following as 

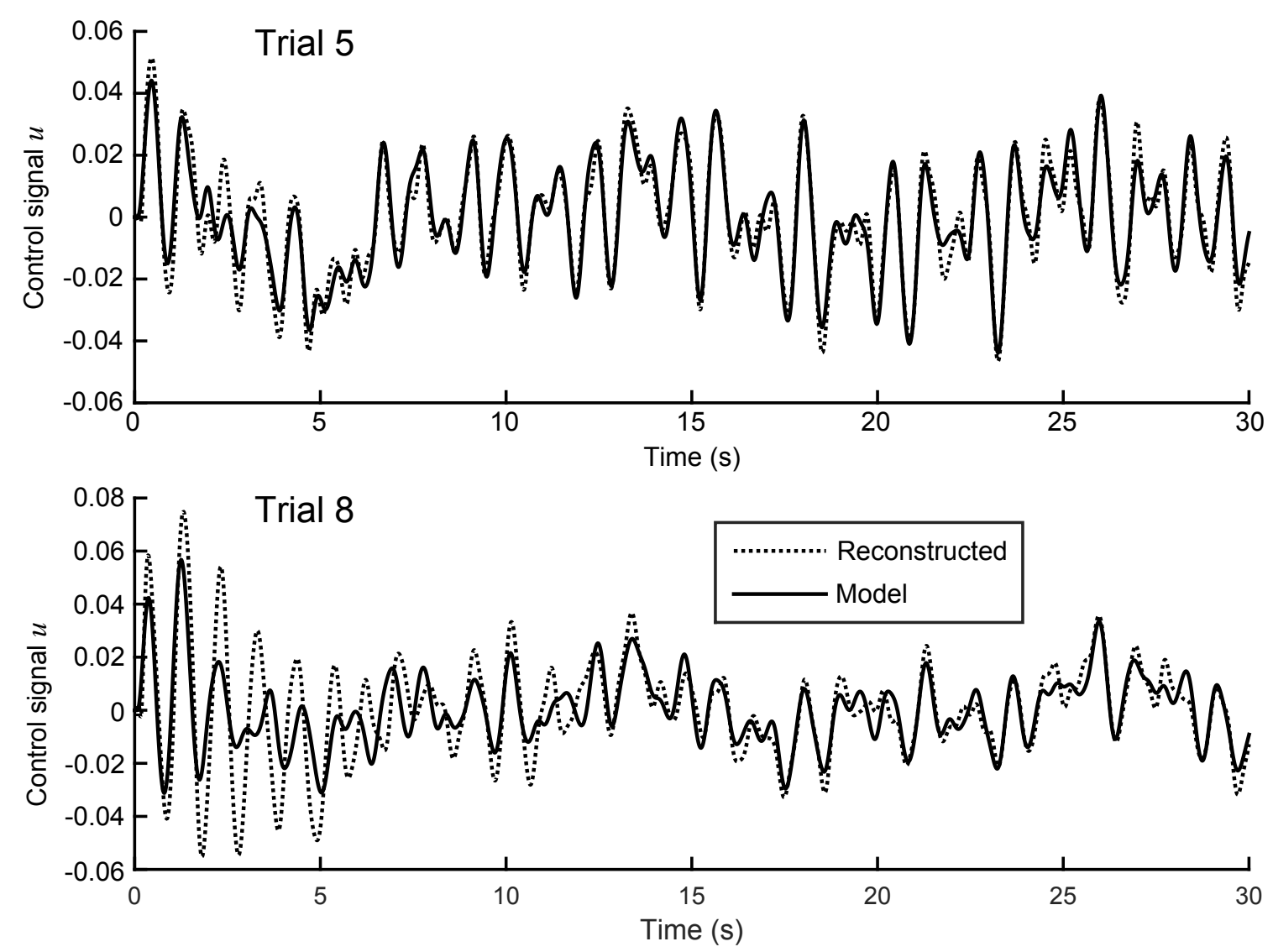

Figure 7. Comparison of first $30 \mathrm{~s}$ of pilot control signal reconstructed from published results of Zaal et al. (2009a) with control signal predicted using new model. The model has been simulated using the separate parameter values identified for each trial, and results are shown for trials 5 and 8.
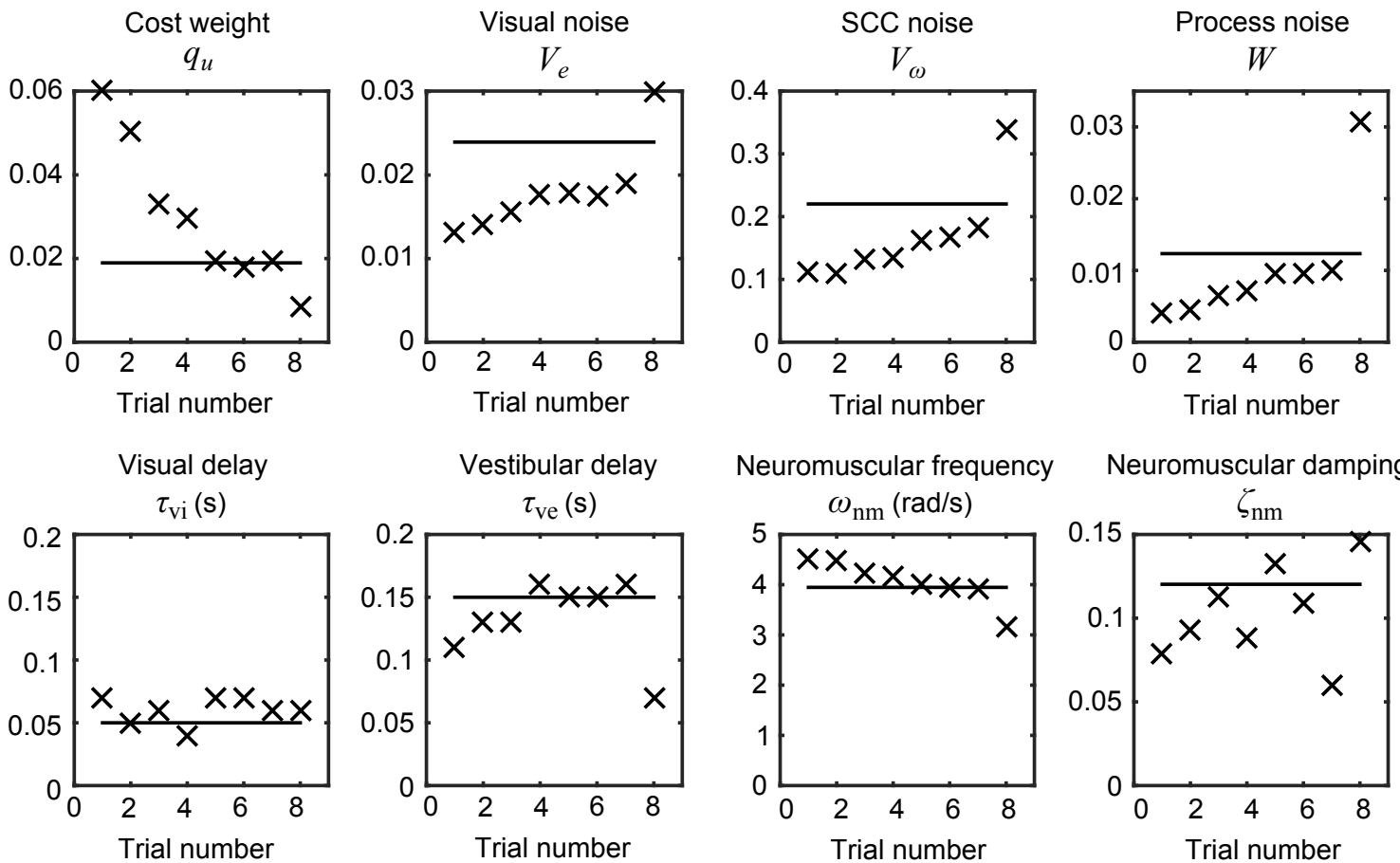

Figure 8. Parameter values identified for the new model to fit each of the trials carried out by Zaal et al. (2009). Separate parameter values identified to fit each individual trial are shown by crosses, the single set of parameter values found to fit the results of all trials is shown by horizontal lines. 
well as disturbance-rejection. Some variations were seen in the identified parameter values across the trials, however a fixed set of parameter values was found to match the results almost as well as separate parameter values identified for each trial. These results have indicated that the new model can predict human control behaviour accurately during a target-following and disturbancerejection task. Work is currently under way to identify and validate the model for driver steering behaviour using results measured in a similar experiment performed in a driving simulator.

\section{REFERENCES}

Bigler, R.S. 2013. Automobile Driver Sensory System Modeling. PhD Thesis. University of Cambridge, Department of Engineering.

Cole D.J., Pick A.J. \& Odhams A. 2006. Predictive and linear quadratic methods for potential application to modelling driver steering control. Vehicle System Dynamics 44(3): 259-284.

Donges E. 1978. A Two-Level Model of Driver Steering Behavior. Human Factors: The Journal of the Human Factors and Ergonomics Society 20(6): 691-707.

Ljung, L. 1999. System Identification: Theory for the User (2nd edition). Upper Saddle River, New Jersey: Prentice Hall.

Pick, A.J. 2004. Neuromuscular Dynamics and the Vehicle Steering Task. PhD Thesis. University of Cambridge, Department of Engineering.

Pick, A.J. \& Cole, D.J. 2007. Dynamic properties of a driver's arms holding a steering wheel. Proceedings of the Institution of Mechanical Engineers, Part D: Journal of Automobile Engineering 221(12): 14751486.

Plöchl, M. \& Edelmann, J. 2007. Driver models in automobile dynamics application. Vehicle System Dynamics 45(7-8): 699-741.

Rodchenko, V., Boris, S., \& White, A. 2000. In-flight estimation of pilots acceleration sensitivity thresholds. Modeling and Simulation Technologies Conference e4292. Reston, Virginia: American Institute of Aeronautics and Astronautics.

Samji, A. \& Reid, L.D. 1992. The detection of low-amplitude yawing motion transients in a flight simulator. IEEE Transactions on Systems, Man, and Cybernetics 22(2): 300-306.

Sharp, R.S. \& Valtetsiotis, V. 2001. Optimal preview car steering control. Vehicle System Dynamics Supplement 35: 101117.

Telban, R.J. \& Cardullo, F. 2005. Motion cueing algorithm development: Human-centered linear and nonlinear approaches. NASA Technical Report.

Valente Pais, A.R., Pool, D.M. et al. 2012. Pitch Motion Perception Thresholds During Passive and Active Tasks. Journal of Guidance, Control, and Dynamics 35(3): 904-918.

Zaal P.M.T., Pool D.M. et al. 2009a. Multimodal Pilot Control Behavior in Combined Target-Following Disturbance-Rejection Tasks. Journal of Guidance, Control, and Dynamics 32(5): 1418-1428.

Zaal, P.M.T., Pool, D.M. et al. 2009b. Modeling Human Multimodal Perception and Control Using Genetic Maximum Likelihood Estimation. Journal of Guidance, Control, and Dynamics 32(4): 1089-1099.

Zaichik, L., Rodchenko, V. et al. 1999. Acceleration perception. Modeling and Simulation Technologies Conference and Exhibit e4334. Reston, Virginia: American Institute of Aeronautics and Astronautics. 